\title{
Juxtaposition of heterochromatic and euchromatic regions by chromosomal translocation mediates a heterochromatic long- range position effect associated with a severe neurological phenotype
}

Palma Finelli ${ }^{1} 2^{*}$, Silvia Maria Sirchia ${ }^{3,4}$, Maura Masciadri ${ }^{1}$, Milena Crippa ${ }^{1}$, Maria Paola Recalcati ${ }^{1}$, Daniela Rusconi ${ }^{1}$, Daniela Giardino ${ }^{1}$, Laura Monti ${ }^{3}$, Francesca Cogliati ${ }^{1}$, Francesca Faravelli ${ }^{5}$, Federica Natacci ${ }^{6}$, Leonardo Zoccante ${ }^{7}$, Bernardo Dalla Bernardina ${ }^{7}$, Silvia Russo ${ }^{1}$ and Lidia Larizza ${ }^{1,3}$

\begin{abstract}
Background: The term "position effect" is used when the expression of a gene is deleteriously affected by an alteration in its chromosomal environment even though the integrity of the protein coding sequences is maintained. We describe a patient affected by epilepsy and severe neurodevelopment delay carrying a balanced translocation $\mathrm{t}(15 ; 16)(\mathrm{p} 11.2 ; \mathrm{q} 12.1) \mathrm{dn}$ that we assume caused a position effect as a result of the accidental juxtaposition of heterochromatin in the euchromatic region.

Results: FISH mapped the translocation breakpoints (bkps) to 15p11.2 within satellite III and the 16q12.1 euchromatic band within the ITFG1 gene. The expression of the genes located on both sides of the translocation were tested by means of real-time PCR and three, all located on der(16), were found to be variously perturbed: the euchromatic gene NETO2/BTCL2 was silenced, whereas VPS35 and SHCBP1, located within the major heterochromatic block of chromosome 16q11.2, were over-expressed. Pyrosequencing and chromatin immunoprecipitation of NETO2/BTCL2 and VPS35 confirmed the expression findings. Interphase FISH analysis showed that der(16) localised to regions occupied by the beta satellite heterochromatic blocks more frequently than der(15).

Conclusions: To the best of our knowledge, this is the first report of a heterochromatic position effect in humans caused by the juxtaposition of euchromatin/heterochromatin as a result of chromosomal rearrangement. The overall results are fully in keeping with the observations in Drosophila and suggest the occurrence of a human heterochromatin position effect associated with the nuclear repositioning of the der(16) and its causative role in the patient's syndromic phenotype.
\end{abstract}

Keywords: Balanced translocation, Heterochromatin, Position effect, Gene expression perturbation, Epigenetic modification

\footnotetext{
* Correspondence: finelli@auxologico.it

'Laboratory of Medical Cytogenetics and Molecular Genetics, Istituto

Auxologico Italiano, Cusano Milanino 20095, Italy

Full list of author information is available at the end of the article
} 


\section{Background}

Patients with syndromic clinical phenotypes include an interesting subset of carriers of de novo balanced chromosomal rearrangements with no apparent loss or gain of genetic material. These abnormalities can be explained by the loss of function of a dose-sensitive gene disrupted by one rearrangement breakpoint [1-3]. The breakpoints of the balanced chromosomal rearrangements associated with a clinical phenotype are often molecularly mapped in an attempt to identify the disease-causing genes affected by the abnormality. Although the rearrangements may lead to the direct disruption of one or two genes, this is not always the case. It has also been shown that breakpoints occur outside the genes themselves and affect their regulation by causing a change in their position within the genome, a phenomenon known as the "position effect" (PE). Investigations of the PE have required intensive experimental effort because of the unique features of each case and the often scarcely defined length of the involved genomic region.

A number of potential mechanisms can be suggested to explain the position effects of chromosomal rearrangements in humans for a review see ref. [4]: 1) the separation of a gene from its enhancer, promoter or locus control region; 2) juxtaposition to an enhancer element from another gene; 3 ) the removal of the longrange insulator or boundary elements; 4) competition with another enhancer; 5) alterations in local chromatin structure; 6) alterations in nuclear organisation [5,6]; and 7) position effect variegation (PEV), when the genes are moved into close proximity to constitutive heterochromatin and their activity become unstable and leads to variegated patches of gene expression.

PEV was first observed in Drosophila and was so named because of the variegated pattern of expression of euchromatic genes transposed to locations near to pericentric heterochromatin as a result of natural or induced genetic rearrangements [7-10]. The chromosomal position effect can spread over distances of $1 \mathrm{Mb}$ or more, and generally reflects a gradient of gene inactivation that is inversely correlated with distance [10], although it can also be affected by the local context of a gene [11].

Some genes are specifically adapted to be expressed exclusively in a heterochromatic context (heterochromatic genes) [9]. They are also influenced by PEV, but their behaviour is opposite to that of genes located in euchromatic chromosomal regions (euchromatic genes). The regulatory implication of genes residing in heterochromatic regions was first evidenced in a pioneering study that linked PEV to euchromatic/heterochromatic rearrangements: the variegation of the light gene, known as Dmel $\backslash l t$, encoding a protein containing a zinc ion binding domain and residing at the heterochromatin side of the rearrangement breakpoint, was enhanced by an increased dose of $\mathrm{Y}$ chromosome constitutive heterochromatin, whereas the variegation of genes located on the euchromatic side of the breakpoint was suppressed [12]. Subsequently, it was found that a number of other heterochromatic genes show similar heterochromatic dependence [13-15], some of which are unique in terms of function and protein coding [16].

Sequencing projects have led to the discovery of hundreds of heterochromatic genes in Drosophila, plants and mammals, but no variegation effects have yet been reported in humans, although they have been observed in transgenic mice bearing incomplete functional gene domains that became inserted into heterochromatic regions [17].

Given the variability of chromosomal rearrangements, rare patients carrying unique chromosomal abnormalities often show single or multiple clinical signs that cannot be assigned to any recognisable syndrome. We here describe the molecular cytogenetic, genetic and epigenetic characterisation of a "private" balanced chromosomal translocation $\mathrm{t}(15 ; 16)(\mathrm{p} 11.2 ; \mathrm{q} 12.1) \mathrm{dn}$ carried by a patient affected by epilepsy and severe neurodevelopment delay. Fluorescence in situ hybridisation (FISH) breakpoint mapping and analyses of the quantitative expression and promoter epigenetic signatures of the genes at or near the breakpoints showed that a simple chromosomal rearrangement may involve multiple genes as a result of a long-range heterochromatic position effect.

\section{Results}

\section{Clinical presentation}

The proband is the first and only daughter of healthy and unrelated parents, and was born in the $39^{\text {th }}$ g.w. after an uncomplicated pregnancy with a birthweight of $3480 \mathrm{~g}\left(50^{\text {th }}\right.$ centile), a length of $50 \mathrm{~cm}\left(50^{\text {th }}\right.$ centile), and a head circumference of $33.5 \mathrm{~cm}$ ( $10^{\text {th }}$ centile). At birth, she was affected by neonatal respiratory distress and hypotonia.

During her first day of life, she experienced brief partial seizures with secondary generalisation that were treated with phenobarbital (PB) but recurred several times a week until she was two months old, when they started appearing as clusters of infantile axial spasms. The spasms disappeared after treatment with valproic acid (VPA), and the baby started to smile and gained control of her head. At the age of six months, the spasms returned and became daily until she was 30 months old, despite the administration of adrenocorticotropin hormone $(\mathrm{ACTH})$ and gamma-vinyl GABA 
(GVG). Interictal electroencephalography (EEG) revealed the presence of frequent paroxysms involving the temporo-parietal regions of both hemispheres, with left predominance in the absence of a hypsarrhythmic pattern.

Subsequently, the spasms became progressively less frequent with VPA treatment, occurring only a few times a month during sleep or upon awakening. They finally disappeared when the patient was five years old, and interictal EEG revealed normal findings during wakefulness and rare focal spikes on the parietal regions only during sleep.

From the age of four years, the patient's clinical picture progressively improved. Now aged 12 years, she can sit alone, has hypotonic-dystonic cerebral palsy, is averbal, and suffers from very severe cognitive impairment, but her relationships are relatively good.

Cerebral MRI at the age of three years revealed a left temporo-parietal focal abnormality suggesting a prenatal insult with micropolygyria.

A physical examination at the age of 10 years showed a length of $120 \mathrm{~cm}\left(<3^{\text {rd }}\right.$ centile), a weight of $20 \mathrm{~kg}(<$ $3^{\text {rd }}$ centile $)$, and a head circumference of $49 \mathrm{~cm}\left(<3^{\text {rd }}\right.$ centile), together with brachyplagiocephaly, wide palpebral fissures with mild lateral ectropion and long eyelashes, thin fingers, and severe thoracic scoliosis.

The patient was originally referred to our laboratory because of suspected Angelman syndrome, which was ruled out as SNRPN methylation analysis showed a biparental pattern and no UBE3A gene mutation was found. Rett syndrome was ruled out by means of mutation screening of the $M e C p 2$ and CDKL5 genes.

\section{Translocation breakpoints analysis}

Karyotype analysis showed a balanced translocation $\mathrm{t}$ $(15 ; 16)(\mathrm{p} 11.2 ; \mathrm{q} 12.1) \mathrm{dn}$.

FISH characterisation narrowed the $15 \mathrm{p}$ breakpoint region to within $15 \mathrm{p} 11.2$ satellite III (D15Z1) on the basis of the fact that probe D15Z1 gave a signal on both derivative chromosomes 15 and 16 (Figures $1 \mathrm{~A}$ and 1D), whereas RPC1-98 C19 and RPC1-21I10 (containing beta satellite sequences) only gave a signal on $\operatorname{der}(16)$ (Figures $1 \mathrm{~A}$ and $1 \mathrm{D})$, as did the rDNA probe RPC5-1174A5 (Figures $1 \mathrm{~B}$ and 1D). As expected, the $\alpha$-satellite probes D15Z3 and D15Z4 gave a signal on the centromere of $\operatorname{der}(15)$ (Figures 1C and 1D).

The $16 \mathrm{q}$ translocation bkp was mapped to $16 \mathrm{q} 12.1$ using a BAC contig and was localised within the ITFG1 gene, which is disrupted: the contiguous BACs showed different hybridisation patterns, with CTD-3056I17 only giving a signal on der(16) (Figure 1E), CTD-2027D24 giving a signal on both derivative chromosomes (Figure $1 F)$, and RP11-474B12 giving a signal only on $\operatorname{der}(15)$ (data not shown). In order to refine the localisation of the $16 \mathrm{q}$ breakpoint, a $7 \mathrm{~kb}$ long-range PCR genomic

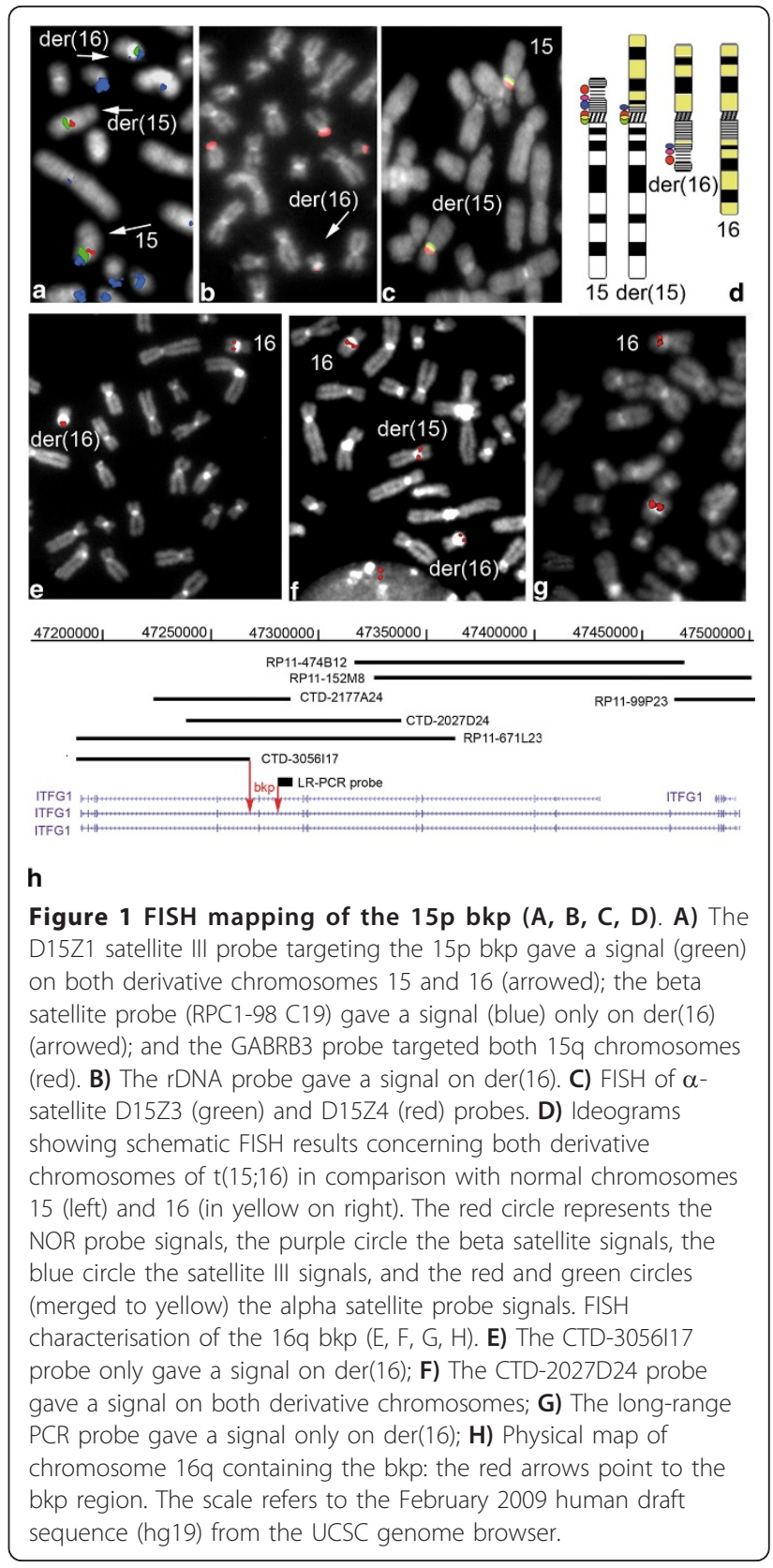

fragment, covering the distal part of IVS12, was hybridised with patient metaphases. This probe only gave a signal on $\operatorname{der}(16)$, the intensity of which was comparable with that of the normal chromosome 16, thus allowing the bkp region to be approximately narrowed to a $12 \mathrm{~kb}$ interval (chr16:47268711-47280772, hg19) (Figure 1G and $1 \mathrm{H})$. All of the probes and the FISH results are shown in Additional file 1: Table S1.

In order to investigate whether the translocation involving the $15 \mathrm{p}$ heterochromatic blocks favoured a relocalisation of the $16 \mathrm{q}$ euchromatic bkp regions in a heterochromatic environment, we performed tripled i-FISH 
experiments using patient lymphoblast cells derived from three different cultures and hybridised with probes D15Z1, GABRB3 and RPC1-98 C19. For each experiment, at least 100 nuclei were analysed by evaluating the co-localisation of the $16 \mathrm{q}$ bkps with regions recognised by the probe RPC1-98 C19, which contains betasatellite DNA.

We observed der(16) co-localisation with heterochromatic regions more frequently than $\operatorname{der}(15)$ co-localisation in all of the experiments (Figure 2).

\section{Array CGH analysis}

The analysis excluded the gain or loss of any genomic region encompassing the $\operatorname{der}(15) / \operatorname{der}(16)$ breakpoints and flanking regions, including the loci monitored for gene expression. It also revealed a loss on chromosome 9 and a gain on the $\mathrm{X}$ chromosome, neither of which were listed in the reference Database of Genomic

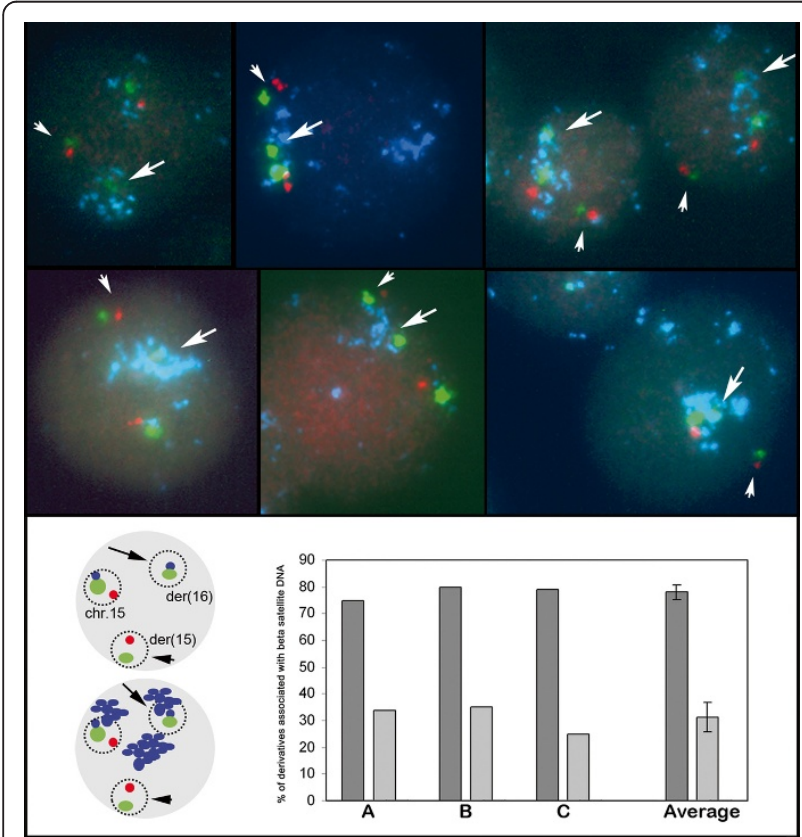

Figure 2 Nuclear position of derivative chromosomes with beta satellite regions. i-FISH (top) performed using D15Z1 (green), GABRB3 (red) and RPC1-98 C19 (blue) containing beta-satellite DNA. The large and small arrows respectively indicate der(16) and der(15). Chromosome 15 is not shown but it occupies the region in which all three hybridization signals are located, with the green signal larger than those identified on the two derivatives. At the bottom left a drawing showing the expected hybridization pattern allowing to recognize the three different chromosomes (der (16), der (15) and chromosome 15) on nucleus. The picture above shows only the expected pattern of hybridization on the three chromosomes in the drawing below is also shown acrocentric regions recognized by the probe containing beta-satellite DNA. At the bottom right graphs $\mathrm{A}$, $B$, and $C$ show the rate of co-localisation of der(16) (dark grey) and der(15) (light grey) to the regions targeted by the beta satellite. The results average of three experiments is shown on the right.
Variants http://projects.tcag.ca/variation, a catalogue of structural variations identified in healthy subjects. In detail, high-resolution a-CGH analysis identified a paternal deletion of at least $28 \mathrm{~kb}$ at 9p24.1 (chr9:6,215,819$6,244,723$, hg19) and a maternal duplication of at least $500 \mathrm{~kb}$ at Xp22.2 (chrX:11,686,237-12,187,337, hg19) (Additional file 2: Figure S1). Two genes, MSL3 and RFMPD4/PDZD10 map to Xp22.2, the latter covering a duplication bkp and hence being hypothetically interrupted. The 9p deletion disrupts the IL33 gene.

\section{$\mathrm{XCl}$ pattern}

In order to verify whether the maternally derived $\mathrm{CNV}$ localised on chromosome $\mathrm{X}$ could be involved in the patient's phenotype, $\mathrm{X}$ chromosome inactivation assay was performed analysing AR and DXS6673E loci. While the AR locus was uninformative, the DXS6673e locus displayed a random $\mathrm{X}$ inactivation in the mother (59:41) whereas the patient showed a preferential $\mathrm{X}$ inactivation of the maternal allele (82:18; Additional file 3: Figure $\mathrm{S} 2$ ). These results indicate no role of this $\mathrm{CNV}$ in the patient phenotype.

\section{Gene expression}

Real-time PCR using primers positioned on the opposite sides of the bkp within ITFG1 IVS12 asses that the amount of the $5^{\prime}$ portion of the transcript was comparable with that of the control and half that of its 3' portion, thus confirming the FISH results (Figure 3).

Given the complex syndromic phenotype of the patient, we tested whether the breakage and juxtaposition of $15 p$ constitutive heterochromatin to the $16 q 12.1$ euchromatic region disturbed the expression of the

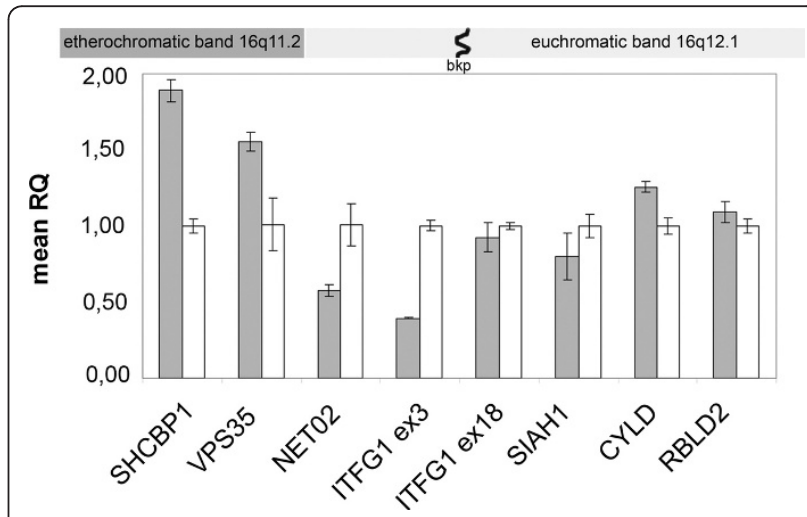

Figure 3 Real-time quantitative PCR showing the relative quantification (RQ) of ITFG1 cDNA amplification product obtained using ex-18 primers, which was found to be half that obtained using ex-3 primers. The expected $\Delta \Delta C t$ ratio is $\cong 1$ when both alleles are expressed, and 0.5 when only one allele is expressed. The genes are indicated on the abscissa and the average of three recorded expression levels on the ordinate. The grey and white bars respectively represent the case and control. 
intact genes flanking the 16q bkp. We first analysed four genes whose expression profiles and functions suggested a possible causative role: VPS35 and NETO2/BTCL2, which respectively map to $16 \mathrm{q} 11.2$ heterochromatic and 16q12.1 euchromatic bands, and are localised 0.5 Mb and $62 \mathrm{~kb}$ from the bkp on $\operatorname{der}(16)$, and SIAH1 and $C B L N 1$, which both map to $16 \mathrm{q} 12.1$ and are localised $1.1 \mathrm{Mb}$ and $2 \mathrm{Mb}$ from the bkp on der(15). Real-time PCR revealed halved expression of NETO2/BTCL2 and the over-expression of VPS35, both located on $\operatorname{der}(16)$, whereas the expression of SIAH1 was comparable with that of the control (Figure 3). The expression of the brain-specific $C B L N 1$ gene was not quantifiable because it was low in all of lymphoblast cell lines.

In order to establish whether other more distant genes were transcriptionally perturbed, we also analysed the expression of the der(16) genes lying at distances from bkp of $1 \mathrm{Mb}$ (SHCBP1), $0.56 \mathrm{Mb}$ (ORC6L), $0.67 \mathrm{Mb}$ (GPT2) and 0.27 Mb (DNA JA2); SHCBP1 and ORC6L are located in the major heterochromatic block of chromosome 16 (16q11.2), and GPT2 and DNA JA2 in the euchromatic band 16q12.1. We also monitored the expression of the following genes located on $\operatorname{der}(15)$ at 16q12.1: ABCC12 (0.9 Mb from the bkp), N4BP1 (1.4 $\mathrm{Mb}), A D C Y 7$ (3 Mb), BRD7 (3.1 Mb), CYLD (3.5 Mb), SALL1 (3.9 Mb) RBL2 (6.2 Mb), HERPUD (9.7 Mb) and CKLF (19 Mb). Only the SHCBP1 gene was expressed at higher levels than the control levels (Figure 3), whereas the expression of most of the genes (DNA JA2, ADCY7, $B R D 7, C Y L D$ and $R B L 2$ ) was comparable with that of the controls (data non shown). In relation to the remaining genes $(O R C 6 L, A B C C 12, N 4 B P 1, S A L L 1$, HERPUD and CKLF), we could not draw any conclusions because of their low level of expression in the lymphoblast cell lines or the variability of expression in the control samples (GPT2).

\section{Epigenetic characterisation of the promoter regions}

It is well known that $\mathrm{H} 3 \mathrm{mK} 9, \mathrm{H} 3 \mathrm{mK} 27$ and $\mathrm{CpG}$ site methylation occurs in silenced gene promoters, whereas the acetylation of histones $\mathrm{H} 3$ and $\mathrm{H} 4$ and $\mathrm{H} 3$ lysine 4 methylation correlate with transcriptionally active genes. In order to assess the epigenetic status of the intact genes flanking both sides of the $16 \mathrm{q}$ breakpoint, lymphoblastoid cell lines from the patient and controls were investigated for their DNA methylation status and chromatin modifications in the regulatory regions of the genes whose expression was disturbed or slightly perturbed. In particular, we tested the promoter regions of the NETO2/BTCL2, SIAH1, CYLD and $R B L 2$ genes.

The promoter methylation analysis was made using a pyrosequencing assay that quantitatively measures the methylation of all $\mathrm{CpG}$ sites. In the normal samples,
NETO2/BTCL2, SIAH1, CYLD and RBL2 were demethylated at all of the analysed $C \mathrm{pG}$ sites; in the patient, and in line with the gene expression results, we found a NETO2 promoter methylation pattern indicating the epigenetic silencing of one allele (mean 50\% methylation) (Figure 4), whereas all of the other gene promoters located on $\operatorname{der}(15)$ (SIAH1, CYLD and RBL2) showed a methylation pattern comparable with that of the normal samples (Figure 4).

We then tested seven different modifications on the histone tails: $\mathrm{H} 3, \mathrm{H} 4$ acetylation, and $\mathrm{H} 3 \mathrm{di}$ - and trimethylation on lysine 4 (associated with an active chromatin state), and $\mathrm{H} 3 \mathrm{di}-$ and tri-methylation on lysine 9 and $\mathrm{H} 3$ di-methylation on lysine 27 , which are specific for transcriptionally silenced chromatin. The ChIP assays showed $\mathrm{H} 3 \mathrm{~K} 9 \mathrm{di}-$ and tri-methylation and $\mathrm{H} 3$ K27 di-methylation in the NETO2/BTCL2 promoter (Additional file 4: Table S2), whereas VPS35 and SIAH1 showed the same qualitative histone code as the control. As VPS35 was up-regulated, we used Real-time PCR on chromatin immunoprecipitates to assess the quantitative changes in the epigenetic modifications related to the trascriptionally competent chromatin, and found increased $\mathrm{H} 4$ acetylation and $\mathrm{H} 3$ tri-methylation on lysine 4 (Figure 5).

\section{Discussion}

We believe that this may be the first description of a human disease involving a heterochromatic PE, by means of which intact euchromatic and heterochromatic genes are respectively silenced or enhanced as a result of euchromatin/heterochromatin juxtaposition to constitutive heterochromatin. Although our molecular analysis could not assay variegation in the classical sense of PEV, at least three genes showed perturbed expression and behaviour equivalent to that of Drosophila genes in the same chromosomal context [8]. As in Drosophila, we found the over-expression of heterochromatic genes (VPS35 and SHCBP1) located at 16q11.2, and the under-expression of the euchromatic gene (NETO2/ $B T C L 2)$ located at $16 q 12.1$. In keeping with the expression results and the findings observed in Drosophila [18], the silenced NETO2/BTCL2 "euchromatic" gene on $\operatorname{der}(16)$ showed promoter DNA methylation and H3 K9 di- and tri-methylation/H3 K27 di-methylation histone modifications, whereas the over-expressed VPS35 "heterochromatic" gene showed an increase in $\mathrm{H} 4$ acetylation and $\mathrm{H} 3$ tri-methylation on lysine 4 in comparison with the control. These findings confirm that these chromatin modifications act as universal markers of active/inactive genes in both the heterochromatic and euchromatic bands [18]. We did not observe any epigenetic silencing of the assayed $\operatorname{der}(15)$ genes SIAH1, $C Y L D$ and $R B L 2$. 


\section{NETO2 CTRL}

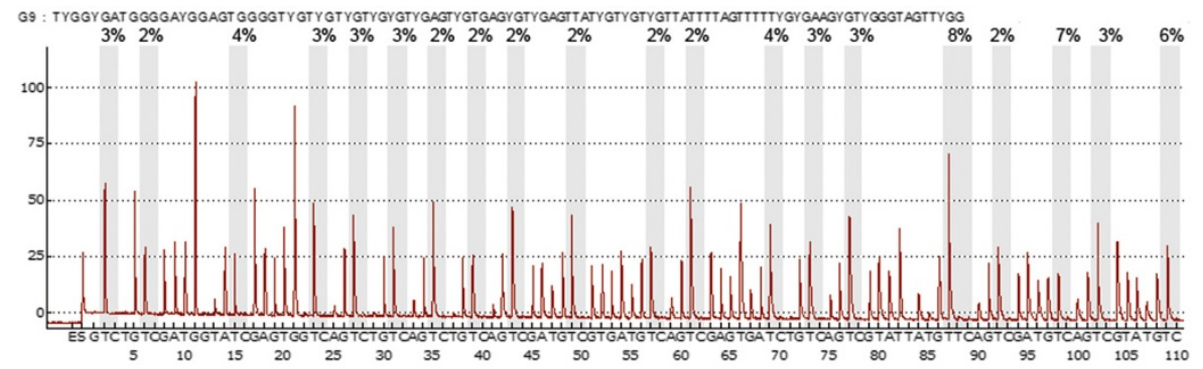

NETO2 PT

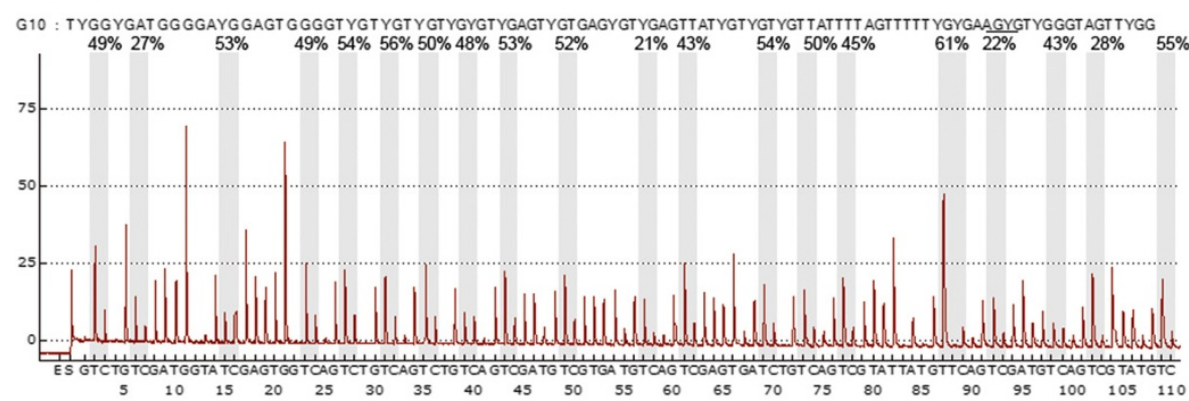

\section{SIAH1 CTRL}

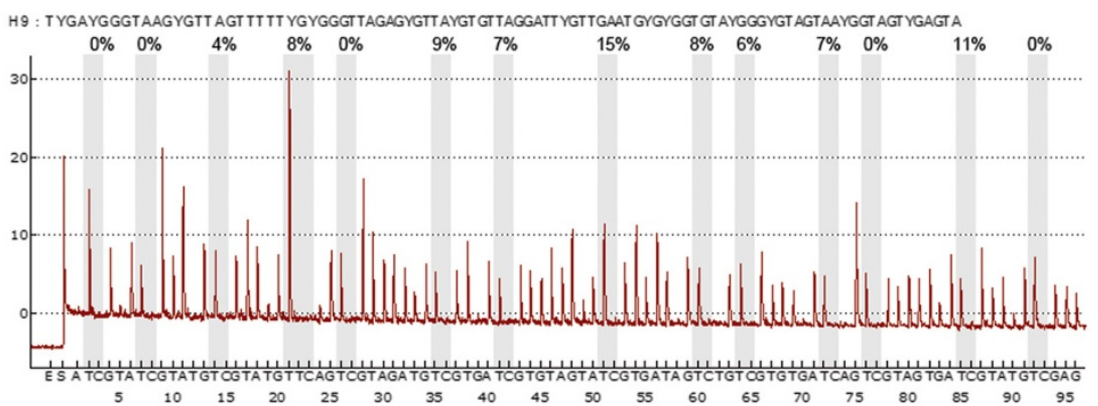

\section{SIAH1 PT}

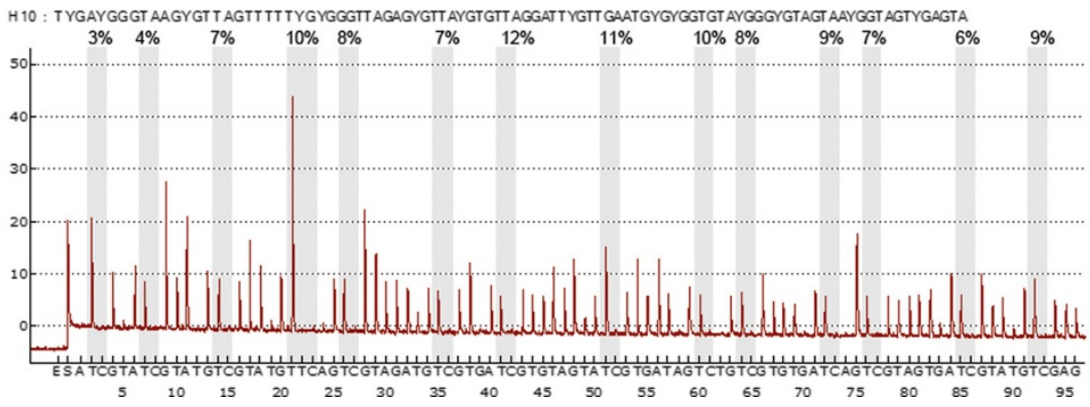

Figure 4 Pyrograms indicating the percentage of methylation of positions $20 \mathrm{CpG}$ and $14 \mathrm{CpG}$ within the NETO2 and SIAH1 promoter regions in the patient (PZ) and normal lymphoblast cell lines (CTRL). The mean methylation percentages are shown in brackets.

The expression profiles and epigenetic findings consistently revealed the presence of an alteration only on one side of the translocation breakpoint, on the der(16) chromosome. Interestingly but reasonably expected, we also observed that der(16), owning chromosome 15 satellites, is more frequently associated with acrocentric chromosomes beta satellite heterochromatic blocks than der(15). In line with the results of recent studies leading 


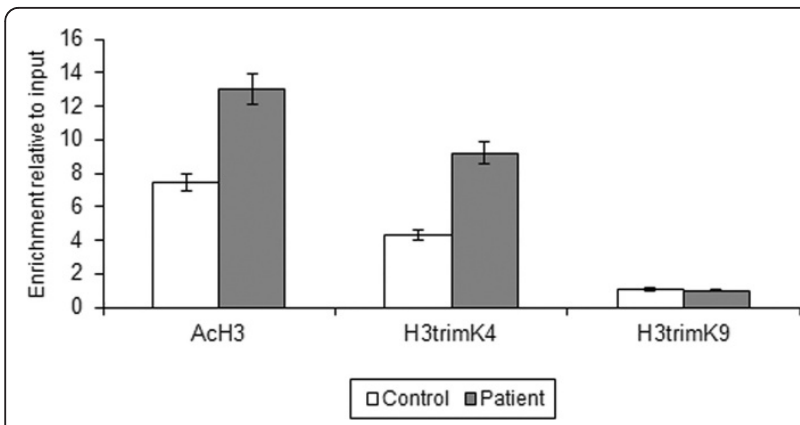

Figure 5 Real-time quantitative PCR of the immunoprecipitated chromatin VPS35 promoter. The results of two independent experiments.

to the conclusion that the reorganisation of chromosomal territories may be an important step in the process of gene silencing [6], our overall findings suggest that the different behaviour of the two derivative chromosomes may be caused by the different nuclear re-localisation of der(16).

Treatment with VPA, acting also as HDAC inhibitor, could modify the epigenetic profile of the patient $[19,20]$, nevertheless the epigenetic signature observed in the derivative chromosomes is coherent with a PE mechanism and not altogether justifiable by the drug treatment.

In terms of genotype-phenotype correlations, our assumption is that most of the observed clinical signs of our patient can be attributed to the de novo balanced translocation. The clinical phenotype of the patient can be interpreted as an effect of the disruption of the ITFG1 gene combined with the heterochromatic PE effect (assuming that the genes in question are dose sensitive).

The 16q translocation bkp disrupts the ITFG1 gene (an inhibitory regulator of PP2A that plays a role in modulating kinase levels in the ATM/ATR signalling pathway), leading to a truncated transcript that is not degraded by nonsense-mediated RNA decay [21]. Moreover, some of the perturbed genes can be plausibly considered causative candidates on the basis of their expression profiles and functions. These include NETO2/BTCL2, which is highly expressed in fetal brain and may play a role in the development and/or maintenance of neuronal circuitry $[22,23]$ as a similar gene in rats encodes a protein that modulates glutamate signalling in the brain by regulating kainate receptor function $[24,25]$. The over-expressed VPS35 is also highly expressed in the brain, and encodes an essential component of the retromer complex [26,27]. The investigation of other plausible candidates (e.g. CBLN1) was precluded by their lack of expression in lymphoblastoid cell lines.
The imbalance in the $\mathrm{X}$ chromosome inherited from the patient mother could contribute to the patient's phenotype as it contains MSL3 gene and RFMPD4/PDZD10 gene, acting respectively as chromatin remodelling/transcription regulating factor $[28,29]$ and as positive regulator of dendritic spine morphogenesis, with a role in the maintenance of excitatory synaptic transmission [30]

Nonetheless, the $\mathrm{X}$ inactivation analysis showed a random $\mathrm{X}$ inactivation in the mother and a preferential maternal $\mathrm{X}$ inactivation in the daughter, excluding a role for this $\mathrm{CNV}$ in the patient's phenotype.

\section{Conclusion}

Our results, which were obtained from genetic and epigenetic studies, suggest the occurrence in human of the $\mathrm{PE}$ due to an euchromatin/constitutive heterochromatin juxtaposition. To our knowledge this is the first report of a human disease involving this kind of $\mathrm{PE}$ mechanism.

Despite our extensive characterisation, the multiple genes that may be involved prevent a full understanding of the pathogenetic mechanism. However, we can reasonably assume that the heterochromatic PE in our case leads to a situation that is comparable with that observed in contiguous gene syndromes. Other carriers of balanced translocations with a breakpoint in heterochromatic sequences need to be similarly studied in order to be able to generalise the contribution of heterochromatic PE to human pathology.

\section{Materials and methods \\ Cytogenetic and FISH analyses}

QFQ-banded metaphases prepared from peripheral blood lymphocytes using standard procedures were analysed, and their karyotype was described in accordance with ISCN (2009) [31]. The probes used for the FISH characterisation of the 15p bkp were the RPC1-98 C19 and RPC1-21I10 clones, provided by Invitrogene (cat. N. RPCI1.C), both containing beta-satellite DNA and targeting 15 p11 and 15 p13 heterochromatic bands [32]; the RP5-1174A5 clone, which contains the DNA/rRNA coding sequences (NOR regions) http://www.biologia.uniba. it/rmc/5-alfoidi/dJ1174A5.html has been selected from RPCI-5 PACs library provided by the YAC Screening Centre (Dibit, HSR, Milan, Italy); the D15Z1 satellite III probe mixed with the GABRB3 probe (Abbott, cat N. 05 J22-015); and the pMC15 (D15Z3) and pTRA25 (D15Z4) clones containing specific chromosome 15 alpha satellite sequences [33] were kindly provided by Prof. Mariano Rocchi, University of Bari. The contiguous BACs, belonging to the RPCI- 11 and the CTD libraries and used for the FISH characterisation of the $16 q \mathrm{bkp}$, were selected using the UCSC Genome Browser (http://genome.ucsc.edu/, hg19) and provided by 
Prof. Mariano Rocchi (RPCI-11 clones) and by Invitrogene (CITB Human D BAC clones, cat. N. 96012D) The $16 \mathrm{p}$ bkp was also characterised by means of a $7 \mathrm{~kb}$ probe covering part of ITFG1 intron 12, which was constructed by means of long-range PCR using the Expand $20 \mathrm{~KB}^{\text {PLUS }}$ PCR System (Roche, cat N. 11811002001) as previously described [34]. The primer pair (fw 5GGCACCATCTTGGCACACTGCAACCTC3- and rev 5-ATTAGGAACCAGGCCGCACGACAGGAG 3-) was designed from the genomic contig NT_010498, and the amplicon sequences (chr16:47,280,772-47,287,772, hg19) were checked by BLAT against the human sequence in order to ensure amplification specifity. Ten nanograms of clone CTD-2027D24 were used as a template. The 7 kb PCR product was run in 1\% low-melting agarose and subsequently purified. All of the BAC/PAC probes were nick-translation labelled with biotin (Roche, cat N. 11093070910), digoxigenin (Roche, cat N. 11093088910) or CY3-dUTP (Amersham, cat N.PAS3022), whereas the PCR product probe was labelled by means of oligopriming (Prime-It Fluor Fluorescence labelling kit, Stratagene, cat. N. 300380). All of the FISH probes were used following the protocols of Lichter and Cremer with minor modifications [35]. Each of the BAC clones was previously tested on chromosomal spreads from normal controls, and only those giving a signal on the chromosomal band indicated by the Genome Browser (UCSC) were used. The specificity of the probe obtained by means of long-range PCR was validated by a FISH experiment that gave a single signal on chromosome 16q11.2. Digital images were obtained using a Leica DMR epifluorescence microscope (Leica Imaging Systems Ltd) equipped with a CCD camera (Cohu Inc). DEAC, FITC, Cy3, and DAPI fluorescence signals were detected using specific filters. The images were recorded, pseudocolored, and merged using QFISH software (Leica Imaging Systems Ltd), and finally edited using Adobe Photoshop CS4 (Adobe Systems).

\section{Array comparative genome hybridisation (CGH) analysis}

For array CGH analysis genomic DNA was extracted from proband's and parent's whole blood using the Dneasy Blood \& Tissue Kit (Sigma-Aldrich, cat. N. NA2020) according to the manufacturer's instructions. Pooled DNA from the peripheral blood of 10 healthy donors (Promega, cat. N. G1521), sex-matched to the samples, was used as a reference. Genome scan was performed by the Human Genome CGH Microarray Kit 244 K (Agilent Technologies, cat. N. G4411B). Briefly, 3 $\mu \mathrm{g}$ of DNA from the test and the normal reference were processed according to the manufacturer's protocol. Images were extracted using Agilent Feature Extraction software 9.1 and analysed by DNA Analytics 4.0 software. A log ratio plot between test and reference genomic DNA was assigned so that aberrations in test DNA copy number at a particular locus are observed as the deviation of the ratio plots from a modal value of 0 . Aberration calls were identified by the ADM-2 algorithm.

\section{$\mathrm{X}$ inactivation analysis}

The X-inactivation pattern was assessed using the Humara Androgen Receptor locus and the DXS6673E locus methylation assay as previously described [36,37]. PCR products were run by capillary electrophoresis and XCI values determined in heterozygous cases using the formula previously reported [38]. This formula calculates the XCI pattern as $(\mathrm{d} 1 / \mathrm{u} 1) /(\mathrm{d} 1 / \mathrm{u} 1+\mathrm{d} 2 / \mathrm{u} 2)$, where $\mathrm{d} 1$ and $\mathrm{d} 2$ represent the two peak areas from the digested sample and $\mathrm{u} 1$ and $\mathrm{u} 2$ are the corresponding areas of the alleles obtained from undigested DNA.

\section{Expression analysis}

Briefly, RNA was extracted from the patient and controls lymphoblast cell lines using Trizol (Gibco BRL. cat N. 15596-018) DNAse-treated (Invitrogene, cat N. 18068-015), and cDNA synthesized using the SupscriptIII Kit (Invitrogen, cat N. 117-52-050) protocol with gene-specific reverse strand primers.

The investigated genes were SHCBP1, VPS35, ORC6L, GPT2, DNA JA2, NETO2/BTCL2, ITFG1, ABCC12, SIAH1, N4BP, CBLN1, ADCY7, BRD7, CYLD, SALL1, $R B L 2, H E R P U D$, and CKLF. The primer pairs designed for each gene, which spanned coding sequences, are listed in Additional file 5: Table S3. Quantitative RTPCR was performed using the comparative threshold cycle method of the Syber-Green $\left(\mathrm{SYBR}^{\circledR}\right.$ Green PCR Master Mix - Applied Biosystem, cat N. 4309155) protocol, as described by Livak and Aarskog and Vedeler $[39,40]$. The RT-PCR and data analyses were carried out using an ABI PRISM 7900HT Sequence Detection System (Applied Biosystem). The estimated expression of all of the genes was computed from three independent retrotranscriptions obtained from three different RNA extractions; the threshold cycle number $(\mathrm{Ct})$ values were obtained from three replicas, and normalised to the endogenous HGPRT control gene. RNA from three healthy human controls (one female and two males) were used as the calibrator sample.

$\mathrm{Ct}$ values were determined for all of the PCR reactions, and a comparative $\mathrm{Ct}$ method was used to calculate the copy numbers of the expressed transcripts. The relative quantity of expressed transcript was calculated as $\mathrm{RQ}=2-(\Delta \Delta \mathrm{Ct} \pm$ standard deviation $[\mathrm{SD}])[39,40]$.

\section{Methylation analysis}

The methylation status of the NETO2/BTCL2, SIAH1, $R B L 2$ and $C Y L D$ promoter regions was analysed by 
means of pyrosequencing technology. The bisulfite conversion of genomic DNA $(1 \mu \mathrm{g})$ was obtained using the EZ DNA methylation kit (Zymo Research, cat N. D5002). After bisulfite treatment, PCR was carried out in a final volume of $50 \mu \mathrm{l}$ with 2.5 units of Promega Go-Taq polymerase (Promega, cat N. M8301). The primers for modified sequences are summarised in Additional file 4: Table S3. The PCR conditions were 45 cycles of $95^{\circ} \mathrm{C}$ for $30 \mathrm{sec}, 52^{\circ} \mathrm{C}$ for $30 \mathrm{sec}$, and $72^{\circ} \mathrm{C}$ for $30 \mathrm{sec}$, followed by $72^{\circ} \mathrm{C}$ for $5 \mathrm{~min} ; 30 \mu \mathrm{l}$ of the PCR product were used for the pyrosequencing assay carried out using the sequencing primers (Figure 3 ).

The pyrosequencing reactions were performed in a PSQ HS 96 System (Biotage, Uppsala, Sweden) by using Pyro Gold Reagent kits (Qiagene, cat. N. 972804). Methylation was quantified using Pyro Q-CpG Software (Biotage), which calculates the ratio of converted C's (T's) to unconverted C's at each CpG site and expresses this as a percentage of methylation. To assess the methylation pattern under normal conditions, we analysed a control lymphoblastoid cell line and different normal DNA from peripheral blood lymphocytes.

\section{Chromatin immunoprecipitation assay (ChIP)}

Chromatin immunoprecipitation was assayed using the chromatin immunoprecipitation assay kit and antiacetyl-histone $\mathrm{H} 3$ (AcH3) (Upstate Biotechnology, cat N. 06-599), anti-acetyl-histone H4 (AcH4) (Upstate Biotechnology, cat N. 06-866), anti-di and -trimethyl-histone H3 Lys4, H3DimK4 (Upstate Biotechnology, cat N. 07-030) and H3TrimK4 (Upstate Biotechnology, cat N. 04-745), anti-di and -trimethyl-histone H3Lys 9, H3DimK9 (Upstate Biotechnology, cat N. 07-441) and H3TrimK9 (Upstate Biotechnology, cat N. 07-442), and anti-dimethyl-histone H3 Lys27 (H3DimK27) (Abcam anti - H3 Trim K27 cat.N. ab6002) antibodies in accordance with the manufacturer's instructions, with minor modifications. Chromatin was immunoprecipitated from $2 \times 10^{6}$ lymphoblastoid cells (per antibody) taken from the patient and control [41].

Quantitative RT-PCR was performed on immunoprecipitated chromatin as previously described in order to verify the quantitative changes in histone modifications on the VPS35 promoter. The primer sequences specific for the VPS35, NETO2/BTCL2, SIAH1, PGK1 and HPRT genes (the last two used as controls) are shown in Additional file 6: Table S4.

\section{Consent}

Written informed consent to the research investigation, which was approved by the Ethical Clinical Research Committee of Istituto Auxologico Italiano, was obtained from the proband's parents.

\section{Additional material}

Additional file 1: Table S1. BAC FISH results.
Additional file 2: Figure S1. Array CGH profile of index case DNA Left
panel) Whole chromosome X array profile. The scatter plot analysis
shows a duplication in Xp22.2 (horizontal shift to the right of 0). Right
panel) Zoomed-in gene view of left panel focussing on a 1.5 Mb
window within Xp22.2 containing the duplication. Each point represents
a single probe. Logz (ratio) was plotted for all of oligonucleotide probes
on the basis of their chromosome positions. The aberration calls
identified by the ADM-2 algorithm (coloured areas) are shown.
Additional file 3: Figure S2. X chromosome inactivation pattern.
DXS6673E analysis. Electropherograms obtained from undigested and
digested (Hhal and Rsa I enzymes) DNA of mother and patient. Both
samples are heterozygous: patient genotype 1-3 (XCI ratio 82:18), mother
genotype 1-2 (59:41).
Additional file 4: Table S2. Chromatin immunoprecipitation assay
results.
Additional file 5: Table S3. Primers used for quantitative PCR.
Additional file 6: Table S4. Primers used for ChIP and methylation
assay.

\section{Abbreviations}

ACTH: Adrenocorticotropin hormone; aCGH: Array Comparative Genomic Hybridization; CNVs: Copy Number Variants; ChIP: Chromatin limmune Precipitation; EEG: Electroencephalography; FISH: Fluorescence in situ Hybridization; GVG: Gamma vinyl GABA(gamma aminobutyric acid); i-FISH: Interphase Fluorescence in situ Hybridization; MRI: Magnetic Resonance Imaging; NOR: Nucleolar Organization Region; PB: Phenobarbital; PE: Position Effect; PEV: Position Effect Variegation; QFQ: Q-banding methods using quinacrine; VPA: Valproic acid; UCSC: University of California Santa Cruz.

\section{Acknowledgements}

The authors would like to thank the patient's family for their collaboration. This study was supported by a Ministry of Health grant "Ricerca Corrente" to Istituto Auxologico Italiano IRCCS (08C604-2006) and Founding "Accordo quadro Università-Regione Lombardia n 17292".

\section{Author details}

${ }^{1}$ Laboratory of Medical Cytogenetics and Molecular Genetics, Istituto Auxologico Italiano, Cusano Milanino 20095, Italy. ²Dept. of Biology and Genetics for Medical Sciences, Università degli Studi di Milano, Milano 20133, Italy. ${ }^{3}$ Dept. of Medicine, Surgery and Dentistry, Università degli Studi di Milano, Milano 20142, Italy. ${ }^{4}$ Pathology Unit, Fondazione IRCCS, Ca' Granda, Ospedale Maggiore Policlinico, Milano 20122, Italy. ${ }^{5}$ Department of Human Genetics,Ospedale Galliera, Genova 16128, Italy. ${ }^{6}$ Clinical Genetics Unit, Department of Obstetrics and Pediatrics, Fondazione IRCCS, Ca' Granda, Ospedale Maggiore Policlinico, Milan 20122, Italy. ${ }^{7}$ Child Neuropsychiatry Unit, Department of Life Science and Reproduction G.B. Rossi Hospital, Università di Verona, Verona 37126, Italy.

\section{Authors' contributions}

PF designed and initiated the study, monitored data collection and analysis for the whole study. SMS, LL and SR contributed to the study design and in the interpretation of data. MPR, PF and DG did conventional cytogenetics and the FISH experiments and interpreted the data. SR and MM did the expression analyses and interpreted the data. MC and DR performed a-CGH analysis and i-FISH experiments and interpreted the data. SMS and LM did the ChIP and Pyrosequencing assays. FC did the SRNPN methylation analysis and the mutation screening of MeCp2 and CDKL5. LZ, B DB, NF, and FF performed clinical investigation at different times and reviewed all clinical records. PF drafted the manuscript. PF, SS and LL took part in critical revision of the manuscript. PF and LL did the final approval of the manuscript. All authors approved the final version of the report.

\section{Competing interests}

The authors declare that they have no competing interests. 
Received: 15 February 2012 Accepted: 4 April 2012

Published: 4 April 2012

\section{References}

1. Kenwrick S, Patterson M, Speer A, Fishbeck K, Davies K: Molecular analysis of the Duchenne muscular dystrophy region using pulsed field gel electrophoresis. Cell 1987, 48:351-357.

2. Greger V, Knoll JH, Wagstaff J, Woolf E, Lieske P, Glatt H, Benn PA, Rosengren SS, Lalande M: Angelman syndrome associated with an inversion of chromosome 15q11.2q24.3. Am J Hum Genet 1997, 60:574-580.

3. Finelli P, Pincelli Al, Russo S, Recalcati MP, Masciadri M, Giardino D, Cavagnini F, Larizza L: Disruption of friend of GATA 2 gene (FOG-2) by a de novo $t(8 ; 10)$ chromosomal translocation is associated with heart defects and gonadal dysgenesis. Clin Genet 2007, 71:195-204.

4. Kleinjan DA, van Heyningen V: Long-range control of gene expression: emerging mechanisms and disruption in disease. Am J Hum Genet 2005, 76:8-32, Review.

5. Cremer T, Cremer M, Dietzel S, Muller S, Solovei I, Fakan S: Chromosome territories - a functional nuclear landscape. Curr Opin Cell Biol 2006, 18:307-316.

6. Harewood L, Schütz F, Boyle S, Perry P, Delorenzi M, Bickmore WA, Reymond $A$ : The effect of translocation-induced nuclear reorganization on gene expression. Genome Res 2010, 20:554-564.

7. Muller HJ: Types of visible variants induced by X-rays in Drosophila. $J$ Genet 1930, 22:229-335.

8. Baker WK: Position-effect variegation. Adv Genet 1968, 14:133-169.

9. Weiler KS, Wakimoto BT: Hetrochromatin and gene expression in Drosophila. Annu Rev Genet 1995, 29:577-605.

10. Wakimoto BT: Beyond the nucleosome: epigenetic aspects of positioneffect variegation in Drosophila. Cell 1998, 93:321-324.

11. Talbert BP, Henikoff S: A reexamination of spreading of position-effect variegation in the white-roughest region of Drosophila melanogaster. Genetics 2000, 154:259-272.

12. Schultz J: Variegation in Drosophila and the inert chromosomal regions. Proc Natl Acad Sci USA 1936, 22:27-33.

13. Wakimoto BT, Hearn MG: The effects of chromosome rearrangements on the expression of heterochromatic genes in chromosome $2 \mathrm{~L}$ of Drosophila melanogaster. Genetics 1990, 125:141-154.

14. Eberl DF, Duyf BJ, Hilliker AJ: The role of heterochromatin in the expression of a heterochromatic gene, the rolled locus of Drosophila melanogaster. Genetics 1993, 134:277-292.

15. Schulze SR, Sinclair DA, Fitzpatrick KA, Honda BM: A genetic and molecular characterization of two proximal heterochromatic genes on chromosome 3 of Drosophila melanogaster. Genetics 2005, 169:2165-2177.

16. Dimitri $\mathrm{P}$, Corradini N, Rossi $F$, Vernì $F$ : The paradox of functional heterochromatin. Bioessays 2005, 27:29-41, Review.

17. Milot E, Strouboulis J, Trimborn T, Wijgerde M, de Boer E, Langeveld A, TanUn K, Vergeer W, Yannoutsos N, Grosveld F, Fraser P: Heterochromatin effects on the frequency and duration of LCR-mediated gene transcription. Cell 1996, 87:105-114.

18. Yasuhara JC, Wakimoto BT: Molecular landscape of modified histones in Drosophila heterochromatic genes and euchromatin-heterochromatin transition zones. PLoS Genet 2008, 4:e16.

19. Göttlicher M, Minucci $S$, Zhu P, Krämer $\mathrm{OH}$, Schimpf A, Giavara $\mathrm{S}$, Sleeman JP, Lo Coco F, Nervi C, Pelicci PG, Heinzel T: Valproic acid defines a novel class of HDAC inhibitors inducing differentiation of transformed cells. EMBO J 2001, 20:6969-6978.

20. Phiel CJ, Zhang F, Huang EY, Guenther MG, Lazar MA, Klein PS: Histone deacetylase is a direct target of valproic acid, a potent anticonvulsant, mood stabilizer, and teratogen. J Biol Chem 2001, 276:36734-36741.

21. McConnell JL, Gomez RJ, McCorvey LR, Law BK, Wadzinski BE: Identification of a PP2A-interacting protein that functions as a negative regulator of phosphatase activity in the ATM/ATR signaling pathway. Oncogene 2007, 26:6021-6030.

22. Stöhr $\mathrm{H}$, Berger $\mathrm{C}$, Fröhlich $\mathrm{S}$, Weber $\mathrm{BH}$ : A novel gene encoding a putative transmembrane protein with two extracellular CUB domains and a low-density lipoprotein class A module: isolation of alternatively spliced isoforms in retina and brain. Gene 2002, 286:223-231.

23. Michishita M, Ikeda T, Nakashiba T, Ogawa M, Tashiro K, Honjo T, Doi K, Itohara S, Endo S: Expression of Btcl2, a novel member of Btcl gene family, during development of the central nervous system. Brain Res Dev Brain Res 2004, 153(1):135-142.

24. Zhang W, St-Gelais F, Grabner CP, Trinidad JC, Sumioka A, MorimotoTomita M, Kim KS, Straub C, Burlingame AL, Howe JR, Tomita S: A transmembrane accessory subunit that modulates kainate-type glutamate receptors. Neuron 2009, 61:385-396.

25. Straub C, Hunt DL, Yamasaki M, Kim KS, Watanabe M, Castillo PE, Tomita S: Distinct functions of kainate receptors in the brain are determined by the auxiliary subunit Neto1. Nat Neurosci 2011, 14:866-873.

26. Zhang P, Yu L, Gao J, Fu Q, Zhao Y, Zheng L, Zhao S: Cloning and characterization of human VPS35 and mouse Vps35 and mapping of VPS35 to human chromosome 16q13-q21. Genomics 2000, 70:253-257.

27. Seaman MN, McCaffery JM, Emr SD: A membrane coat complex essential for endosome-to-Golgi retrograde transport in yeast. J Cell Biol 1998, 142:665-681.

28. Smith ER, Cayrou C, Huang R, Lane WS, Côté J, Lucchesi JC: A human protein complex homologous to the Drosophila MSL complex is responsible for the majority of histone $\mathrm{H} 4$ acetylation at lysine $16 . \mathrm{Mo}$ Cell Biol 2005, 25:9175-9188.

29. Sharma GG, So S, Gupta A, Kumar R, Cayrou C, Avvakumov N, Bhadra U, Pandita RK, Porteus MH, Chen DJ, Cote J, Pandita TK: MOF and histone H4 acetylation at lysine 16 are critical for DNA damage response and double-strand break repair. Mol Cell Biol 2010, 30:3582-3595.

30. Lee HW, Choi J, Shin H, Kim K, Yang J, Na M, Choi SY, Kang GB, Eom SH, Kim H, Kim E: Preso, a novel PSD-95-interacting FERM and PDZ domain protein that regulates dendritic spine morphogenesis. J Neurossci 2008, 28:14546-14556

31. Shaffer LG, Slovak ML, Campbell LJ: ISCN An International System for Human Cytogenetic Nomenclature: S Basel: Karger; 2009

32. Piccini I, Ballarati L, Bassi C, Rocchi M, Marozzi A, Ginelli E, Meneveri R: The structure of duplications on human acrocentric chromosome short arms derived by the analysis of 15p. Hum Genet 2001, 108:467-477.

33. Finelli $P$, Antonacci $R$, Marzella R, Lonoce A, Archidiacono A, Rocchi M: Structural organization of multiple alphoid subsets coexisting on human chromosomes 1, 4, 5, 7, 9, 15, 18, and 19. Genomics 1996, 38:325-330.

34. Rauch A, Schellmoser S, Kraus C, Dörr HG, Trautmann U, Altherr MR, Pfeiffer RA, Reis A: First known microdeletion within the Wolf-Hirschhorn syndrome critical region refines genotype-phenotype correlation. Am J Med Genet 2001, 99:338-342.

35. Lichter $\mathrm{P}$, Cremer T: Chromosome analysis by non-isotopic in situ hybridization. In Human Cytogen A Practical Approach. Edited by: Rooney DE, Czipolkowski BH. Oxford: University Press; 1992:157-192.

36. Allen RC, Zoghby HY, Mosely AB, Rosenblatt HM, Belmon JW: Methylation of Hpall and Hhal sites near the polymorphic CAG repeat in the human androgen-receptor gene correlates with $\mathrm{X}$ chromosome inactivation. Am J Hum Genet 1992, 51:1229-1239.

37. Beever $\mathrm{CL}$, Sthephenson MD, Penaherrera MS, Jiang RH, Kalousek DK, Hayden M, Field L, Brown CJ, Robinson WP: Skewed X-chromosome inactivation is associated with trisomy in women ascertained on the basis of recurrent spontaneous abortion or chromosomally abnormal pregnancies. Am J Hum Genet 2003, 72:399-407.

38. Hatakeyama C, Anderson CL, Beever CL, Penaherrera MS, Brown CJ: The dynamics of X-inactivation skewing as women age. Clin Genet 2004 66:327-332.

39. Livak KJ: Comparitive Ct method ABI Prism 7700 Sequence Detection System. User Bulletin No.2. PE Applied Biosystem 1997.

40. Aarskog NK, Vedeler CA: Real-time quantitative polymerase chain reaction. A new method that detects both the peripheral myelin protein 22 duplication in Charcot-Marie-Tooth type $1 \mathrm{~A}$ disease and the peripheral myelin protein 22 deletion in hereditary neuropathy with liability to pressure palsies. Hum Genet 2000, 107:494-498.

41. Sirchia SM, Ramoscelli L, Grati FR, Barbera F, Coradini D, Rossella F, Porta G, Lesma E, Ruggeri A, Radice P, Simoni G, Miozzo M: Loss of the inactive X chromosome and replication of the active $\mathrm{X}$ in BRCA1-defective and wild-type breast cancer cells. Cancer Res 2005, 65:2139-2146.

doi:10.1186/1755-8166-5-16

Cite this article as: Finelli et al: Juxtaposition of heterochromatic and euchromatic regions by chromosomal translocation mediates a heterochromatic long-range position effect associated with a severe neurological phenotype. Molecular Cytogenetics 2012 5:16. 